\title{
Crecimiento y características de canal en corderos Pelibuey puros y cruzados F1 con razas Dorper y Katahdin en confinamiento
}

\author{
Growth and carcass traits in pure Pelibuey lambs and crosses F1 with Dorper \\ and Katahdin breeds in confinement \\ U Macías-Cruz ${ }^{\mathrm{a}}$, FD Álvarez-Valenzuela ${ }^{\mathrm{a}}$, J Rodríguez-García ${ }^{\mathrm{a}}$, A Correa-Calderóna, \\ NG Torrentera-Olivera ${ }^{a}$, L Molina-Ramírez ${ }^{b}$, L Avendaño-Reyes ${ }^{{ }^{*}}$ \\ anstituto de Ciencias Agrícolas, Universidad Autónoma de Baja California, Baja California, México. \\ bBachillerato Tecnológico Agropecuario No. 41, Baja California, México.
}

\begin{abstract}
SUMMARY
The aim of this study was to evaluate feedlot performance and carcass characteristics of 36 male and female lambs from the genotypes pure Pelibuey, Dorper x Pelibuey, and Katahdin x Pelibuey under desert conditions in northwestern Mexico. Lambs were slaughtered for carcass evaluation after $85 \mathrm{~d}$ of a productive performance test. Dorper x Pelibuey lambs had higher $(\mathrm{P}<0.05)$ daily weight gain and feed intake than the other genotypes. However, there were no differences $(\mathrm{P}>0.05)$ among the three genotypes in carcass yield, Longissimus dorsi muscle area and back-fat thickness. Males presented higher $(\mathrm{P}<0.01)$ daily weight gain and lower $(\mathrm{P}<0.01)$ feed conversion than females. Hot and cold carcasses were heavier $(\mathrm{P}<0.05)$ in males than in females, but carcass yield and back-fat thickness were similar $(\mathrm{P}>0.05)$ between sexes. Yield and total weight of primary cuts were also similar $(\mathrm{P}>0.05)$ among genotypes and sexes. These findings suggest that the Dorper breed can be used in crossbreeding schemes to improve mutton production in arid zones, like in northwestern Mexico.
\end{abstract}

Palabras clave: ovinos de pelo, cruzamientos, crecimiento, canales, estrés calórico.

Key words: sheep, crossbreeding, growth, carcasses, heat stress.

\section{INTRODUCCIÓN}

Las condiciones climáticas extremas predominantes en las zonas desérticas de todo el mundo han sido factores que afectan el desarrollo de la actividad agropecuaria en estas regiones debido al estrés que se presenta en el animal. Pocas razas de las diferentes especies de animales domesticados son capaces de sobrevivir y, más aún, de producir eficientemente bajo estas condiciones. Normalmente, este clima exige a las diferentes especies un máximo de disponibilidad de energía para mantener la homeostasis en su cuerpo, lo cual limita la producción (Marai y col 2007). Algunas razas de ovinos y cabras se encuentran ampliamente distribuidas bajo diferentes climas y han demostrado sobrevivir y producir en condiciones ambientales donde para el ganado sería imposible (El Khidir y col 1998).

En el noroeste de México, donde las condiciones climáticas son típicamente desérticas, los ovinos de pelo de raza Pelibuey han sido adoptados por los productores para la producción de corderos en sus explotaciones. Bajo

Aceptado: 16.06.2010.

* Instituto de Ciencias Agrícolas, UABC, carretera Delta S/N, Ejido Nuevo León, CP. 21705, México; lar62@ hotmail.com estas condiciones, esta raza ha demostrado una gran capacidad reproductiva, rusticidad y adaptación, colaborando en mejorar la eficiencia productiva de los rebaños debido a su reducido manejo y menores costos de producción (Avendaño y col 2004). Sin embargo, los corderos Pelibuey al nacimiento presentan pesos bajos; a su vez, su crecimiento y calidad de la canal son inferiores a las razas de lana o cárnicas al ser clasificada como una raza ligera (Gutiérrez y col 2005). Esta situación ha provocado recientemente que ovinocultores de la región incorporen a sus rebaños sementales de razas especializadas en producción de carne para realizar esquemas de cruzamientos, tales como Dorper y Katahdin. Los ovinos Dorper bajo las condiciones del desierto de Sudáfrica han demostrado excelente adaptación basada en su nula estacionalidad a través del año y gran velocidad de crecimiento (Cloete y col 2000). Por su parte, la raza Katahdin desarrollada en el sur de Estados Unidos se ha caracterizado como de buen desarrollo productivo y reproductivo en condiciones tropicales y áridas (Burke y Apple 2007). Se ha observado que los cruzamientos entre hembras Pelibuey y sementales Dorper o Katahdin producen corderos para el abasto que presentan tasas de crecimiento superiores a los Pelibuey puros, así como buena adaptación en climas áridos (Avendaño y col 2004). En otros estudios realizados en climas templados (Gutiérrez y col 2005) o tropicales (Bores y col 2002, Canton y 
col 2009 ${ }^{a}$ ) también se ha observado una superioridad en crecimiento de corderos Pelibuey cruzados con alguna raza cárnica, de lana o de pelo comparados a los puros, lo cual es atribuido al efecto de heterosis y al más rápido grado de madurez que alcanzan los corderos cruzados. Sin embargo, hace falta generar más información del crecimiento y características de la canal de corderos de pelo cruzados con razas cárnicas, bajo condiciones desérticas del noroeste de México, ya que el ambiente puede ser un factor determinante en la utilización de la energía disponible en la ración y consecuentemente en el desarrollo del animal. Por lo tanto, el objetivo de este estudio fue evaluar el comportamiento productivo en corral y las características de canal en corderos Pelibuey puros y sus cruzas F1 con Dorper y Katahdin bajo un clima desértico.

\section{MATERIAL Y MÉTODOS}

El estudio se realizó en la Unidad Experimental Ovina del Instituto de Ciencias Agrícolas de la Universidad Autónoma de Baja California (UABC), que se encuentra ubicada en el Valle de Mexicali, Estado de Baja California, en el noroeste de México. Las condiciones climáticas predominantes en esta región son clasificadas como Desierto de Sonora, que se caracteriza por ser un clima extremadamente seco y caliente, con temperaturas máximas en verano $\left(50^{\circ} \mathrm{C}\right) \mathrm{y}$ mínimas durante invierno $\left(0^{\circ} \mathrm{C}\right)$. La precipitación media anual es de $80 \mathrm{~mm}$, siendo entre los meses de noviembre y diciembre donde mayormente se concentra el período de lluvias (García 1987).

\section{ANIMALES Y MANEJO}

Todos los procedimientos desarrollados sobre los animales experimentales se realizaron en base a las normas oficiales mexicanas ${ }^{1}$. Un total de 50 corderos nacidos en noviembre del 2006 producto de un esquema de cruzamientos entre 60 hembras de raza Pelibuey y tres machos Dorper, Katahdin y Pelibuey (uno de cada raza) fueron destetados cuando alcanzaron una edad de $90 \mathrm{~d}$. Al destete, a estos animales se les aplicaron vía intramuscular vitaminas (A, D y E) y antiparasitario. Posteriormente se seleccionaron 36 corderos, 12 de cada cruzamiento (seis machos y seis hembras): Pelibuey puro $(\mathrm{Pb})$, Dorper $\mathrm{x}$ Pelibuey (DrX) y Katahdin x Pelibuey $(\mathrm{KaX})$, para realizar el experimento. Como los corderos presentaban una edad variable entre los 129 y 136 d al momento de iniciar la prueba, el peso vivo (PV) inicial se ajustó a $133 \mathrm{~d}$ de edad (Notter y col 1975). Las medias de los PV iniciales

1 Secretaría de Economía de México. 1997. Dirección General de Normas: Catálogo de normas oficiales mexicanas. NOM-051ZOO-1995 (trato humanitario en la movilización de animales) y NOM-033-ZOO-1995 (sacrificio humanitario de los animales domésticos y salvajes en México), http://www.economia-noms.gob. $\mathrm{mx} /$ noms/inicio.do fueron $18,1 \pm 0,73,22,6 \pm 0,73$ y 19,2 $\pm 0,73 \mathrm{~kg}$ para $\mathrm{Pb}$, DrX y KaX, respectivamente. El PV inicial para todos los machos y hembras fue $21,1 \pm 0,59$ y $18,3 \pm 0,59 \mathrm{~kg}$, respectivamente. Los corderos se alojaron en parejas de similar genotipo y sexo en corrales provistos de bebederos, comederos y sombra. La prueba de comportamiento productivo tuvo una duración de 100 d, 15 d de adaptación y 85 d de período experimental. La alimentación durante este período consistió de dos dietas (cuadro 1): a) dieta de iniciación, ofrecida los primeros 32 d (19,6\% PC y 2,65 $\mathrm{Mcal} / \mathrm{kg}$ de EM) y b) dieta de finalización, ofrecida por el resto del experimento (16,4\% PC y $2,73 \mathrm{Mcal} / \mathrm{kg}$ de EM). Todos los días se retiró el alimento rechazado de un día anterior para poder ofrecer alimento fresco a razón de dos veces por día (8:00 y 16:00 h). Además, los corderos fueron pesados cada $18 \mathrm{~d}$ hasta finalizar el experimento para ajustar el alimento ofrecido por día a 4,5\% de su PV. Diariamente, el alimento rechazado y ofrecido se pesó para estimar el consumo diario de alimento.

Una vez que se finalizó la prueba de comportamiento productivo, los corderos de los tres genotipos fueron conducidos a la planta faenadora universitaria y sacrificados por el método de degüello sin previa insensibilización. Previo al sacrificio ( 24 h), se les retiró el agua y el alimento. La piel, cabeza y órganos viscerales fueron retirados de cada

Cuadro 1. Ingredientes y composición química de las dietas utilizadas para la alimentación de los corderos durante el período de engorda.

Ingredients and chemical composition of diets used to feed the lambs during the fattening period.

\begin{tabular}{lrc}
\hline & \multicolumn{2}{c}{ Dietas } \\
\cline { 2 - 3 } Ingredientes (g/kg de alimento) & Iniciación & Finalización \\
\hline Grano de trigo & 417,7 & 533,7 \\
Heno de alfalfa & 313,3 & 266,9 \\
Harina de soya & 156,6 & 106,7 \\
Paja de trigo & 52,2 & 53,4 \\
Melaza & 52,2 & 31,9 \\
Sal común en grano & 4,7 & 4,8 \\
Piedra caliza & 3,3 & 2,5 \\
\hline
\end{tabular}

Composición química (\% en base fresca)

\begin{tabular}{lrr}
\hline Materia seca & 93,8 & 93,6 \\
Materia orgánica & 85,3 & 85,4 \\
Proteína cruda & 19,6 & 16,4 \\
Extracto etéreo & 1,4 & 1,1 \\
Fibra cruda & 14,8 & 13,2 \\
Fibra detergente neutral & 17,5 & 15,1 \\
Cenizas & 8,5 & 8,2 \\
Energía metabolizable (Mcal/kg) & 2,6 & 2,7 \\
\hline
\end{tabular}


canal y su peso fue registrado de forma separada. A su vez, fue medido el peso de la canal caliente. Posteriormente, las canales se refrigeraron durante $24 \mathrm{~h} \mathrm{a} 4^{\circ} \mathrm{C}$ para determinar el peso de la canal fría y la longitud de la canal, la cual se estimó midiendo la distancia desde la última vértebra cervical hasta la última vértebra sacra. Las canales fueron cortadas a lo largo de la línea media dorsal con una sierra eléctrica. El lado derecho de cada canal fue trozado entre la 12 y $13^{\text {va }}$ costilla para medir espesor de grasa dorsal y área del músculo Longissimus dorsi usando una cuadrícula de puntos $(64 \mathrm{~mm})$. Finalmente, se realizó un despiece de cada canal para obtener el peso del cuello y cortes primarios (lomo, costillares, paletas y piernas). La suma de los pesos de los cortes primarios fue considerada como peso total de cortes primarios (PTCP), el cual a su vez fue expresado como porcentaje del peso de la canal fría para determinar el rendimiento en cortes primarios (RCP). El peso de piernas, paletas, lomo y costillas fue expresado como porcentaje del peso de la canal fría, mientras que el peso de la canal caliente fue expresado como porcentaje del peso al sacrificio.

\section{CONDICIONES CLIMÁTICAS}

De la Estación Climatológica Experimental de la UABC se colectó la información referente a las condiciones climáticas (temperatura ambiental, T; y humedad relativa, HR) registradas diariamente durante el desarrollo del experimento. A partir de esta información, se calculó el índice de temperatura-humedad (ITH) con la ecuación propuesta para ovinos (Kelly y Bond 1971):

$$
\mathrm{ITH}=\mathrm{T}-\{[0,55 *(1-\mathrm{HR})] *(\mathrm{~T}-14,4)\}
$$

El ITH sirve para determinar el grado de estrés calórico al cual está sometido un animal bajo condiciones ambientales.

\section{VARIABLES DE ESTUDIO}

Las variables de estudio evaluadas fueron peso final (peso registrado al finalizar la prueba de comportamiento productivo), consumo diario de alimento, ganancia diaria de peso, conversión alimenticia, longitud de la canal, espesor de grasa dorsal y área del músculo Longissimus dorsi. Además se analizó peso de canal caliente, de canal fría, de cabeza, de piel, de órganos viscerales y del total de cortes primarios y se calculó el porcentaje de cada corte primario (en base al peso de canal fría), de rendimiento en canal y del total de cortes primarios.

\section{ANÁLISIS ESTADÍSTICO}

Las variables fueron sometidas a un análisis de varianza utilizando un diseño completamente al azar con un arreglo factorial 2 × 3, donde el modelo incluyó el efecto fijo de genotipo ( $\mathrm{Pb}, \mathrm{DrX}$ y $\mathrm{KaX}$ ) y sexo (macho y hembra), así como su posible interacción. El peso ajustado a 133 d se incluyó como covariable en el modelo, mientras que el peso final fue considerado como una covariable para peso de canal caliente y canal fría. Las comparaciones de medias fueron hechas con la prueba " $t$ " a una $\alpha=0,05$. $\mathrm{El}$ análisis estadístico fue desarrollado con el paquete estadístico SAS versión 9,12 para Windows (SAS Institute, Cary, NC, USA), utilizando el procedimiento PROC GLM (SAS 2004). Los resultados para cada variable de estudio se presentan como medias \pm error estándar.

\section{RESULTADOS}

La temperatura ambiental e ITH variaron entre los meses de estudio (cuadro 2), tendiendo a incrementarse a medida que avanzó el desarrollo del experimento. En promedio (máximos y mínimos), la temperatura, la humedad relativa y el ITH en el período experimental fueron de $25,4{ }^{\circ} \mathrm{C}\left(16,7\right.$ en abril a $36,0{ }^{\circ} \mathrm{C}$ en junio), $30,7 \%$ (9,5 en mayo a $50,7 \%$ en abril) y 22,7 unidades (15,7 en abril a 28,5 unidades en junio), respectivamente. En general, durante los tres meses de estudio se registraron días con ITH superior a las 23 unidades, siendo en mayo y junio donde se observó mayor cantidad de días con estos índices de estrés calórico. Tanto en mayo como en junio la temperatura e ITH presentaron valores promedios de 28,5 y $33,0^{\circ} \mathrm{C}$ y de 23,0 y 25,8 unidades, respectivamente.

Cuadro 2. Condiciones climáticas e índice de temperatura-humedad predominantes durante el desarrollo del experimento*.

Climatic conditions and temperature-humidity index during the experiment.

\begin{tabular}{|c|c|c|c|c|c|c|c|c|c|}
\hline & \multicolumn{3}{|c|}{ Mínimas } & \multicolumn{3}{|c|}{ Máximas } & \multicolumn{3}{|c|}{ Promedios } \\
\hline & $\mathrm{T}$ & $\mathrm{H}$ & ITH & $\mathrm{T}$ & $\mathrm{H}$ & ITH & $\mathrm{T}$ & $\mathrm{H}$ & ITH \\
\hline Abril & 16,7 & 17,2 & 15,7 & 30,0 & 50,7 & 23,1 & 23,7 & 33,0 & 19,8 \\
\hline Mayo & 22,4 & 9,5 & 18,9 & 33,1 & 49,9 & 24,1 & 28,5 & 28,1 & 22,5 \\
\hline Junio & 25,4 & 17,2 & 20,4 & 36,0 & 39,9 & 28,5 & 33,0 & 31,1 & 25,8 \\
\hline
\end{tabular}

\footnotetext{
* $\mathrm{T}=$ Temperatura ambiental, $\mathrm{HR}=$ Humedad relativa, $\mathrm{ITH}=$ Índice de temperatura-humedad.
} 
La interacción genotipo x sexo fue estadísticamente significativa $(\mathrm{P}<0,05)$ sólo para peso final. $\mathrm{El}$ peso final entre hembras $\mathrm{KaX}(34,9 \pm 1,1 \mathrm{~kg})$ y $\mathrm{Pb}(32,3 \pm 1,1 \mathrm{~kg})$ fue estadísticamente similar $(\mathrm{P}>0,05)$, pero menor $(\mathrm{P}<0,05)$ al observado en los machos de los tres genotipos $(38,8 \pm 1,1,39,3 \pm 1,1$ y 37,2 $\pm 1,1 \mathrm{~kg}$ para DrX, KaX y $\mathrm{Pb}$, respectivamente) y las hembras $\operatorname{DrX}(38,9 \pm 1,1 \mathrm{~kg})$. Además, entre los machos y las hembras DrX también se observaron pesos finales similares $(\mathrm{P}>0,05)$.

Los animales estudiados presentaron en promedio $206 \pm 10 \mathrm{~g}$ de ganancia diaria de peso, 1,3 $\pm 0,1 \mathrm{~kg}$ de alimento consumido por día y una conversión alimenticia de 6,6 $\pm 0,3 \mathrm{~kg}$ (cuadro 3). Los corderos DrX ganaron 16 y $25 \%$ más peso por día $(\mathrm{P}<0,05)$ que $\operatorname{los} \mathrm{KaX}$ y $\mathrm{Pb}$, respectivamente; asimismo, consumieron 16 y $18 \%$ más alimento por día $(\mathrm{P}<0,05)$, respectivamente. Los corderos de los tres genotipos consumieron cantidades similares $(\mathrm{P}>0,05)$ de alimento para incrementar un kilogramo de su PV. Por otra parte, se observó que la ganancia diaria de peso y el consumo diario de alimento fue mayor $(\mathrm{P}<0,01)$ en 32 y 14\%, respectivamente, en machos que en hembras. Adicionalmente, la conversión alimenticia en machos $(5,9 \pm 0,2)$ fue menor $(\mathrm{P}<0,05)$ que en hembras $(7,3 \pm 0,2)$.

El genotipo no afectó significativamente $(\mathrm{P}>0,05)$ las medias de rendimiento en canal, peso de la cabeza, peso de los órganos viscerales, área del músculo Longissimus dorsi y espesor de grasa dorsal (cuadro 4). Sin embargo, el peso de la canal caliente $(20,1 \pm 0,6$ vs $18,3 \pm 0,6 \mathrm{~kg})$ y canal fría $(18,9 \pm 0,6 \mathrm{vs} 16,6 \pm 0,6 \mathrm{~kg})$ fue mayor $(\mathrm{P}<0,01)$ en corderos $\mathrm{DrX}$ que en $\mathrm{Pb}$. Las canales de corderos $\mathrm{KaX}$ presentaron pesos semejantes $(\mathrm{P}>0,05)$ a los otros dos genotipos estudiados. En relación a la longitud de la canal, las canales fueron más cortas en el genotipo KaX por $5 \mathrm{~cm}(\mathrm{P}<0,01)$ que las del genotipo $\operatorname{DrX}(63,0 \pm 1,3$ vs 58,0 $\pm 1,3)$, pero de tamaño similar $(\mathrm{P}>0,05)$ a las del genotipo $\mathrm{Pb}$. Las pieles de los corderos cruzados pesaron en promedio $350 \mathrm{~g}$ más $(\mathrm{P}<0,01)$ que las de corderos puros $\mathrm{Pb}$. No obstante, el sexo resultó significativo $(\mathrm{P}<0,05)$ sobre las características de canal medidas, con excepción de las variables $(\mathrm{P}>0,05)$ rendimiento en canal, área del músculo Longissimus dorsi y espesor de grasa dorsal. En los machos se observaron canales más largas $(\mathrm{P}<0,01 ; 62,3 \pm 1,0$ vs $58,7 \pm 1,0 \mathrm{~cm})$ y pesadas $(\mathrm{P}<0,05 ; 20,0 \pm 0,5$ vs $18,2 \pm 0,5 \mathrm{~kg}$ para canal caliente y $18,81 \pm 0,5$ vs $16,54 \pm 0,5 \mathrm{~kg}$ para canal fría), así como valores superiores $(\mathrm{P}<0,05)$ en el peso de cabeza, piel y órganos viscerales en relación a las hembras.

En cuanto a los resultados de cortes primarios (cuadro 5) se observó que el genotipo sólo influyó $(\mathrm{P}<0,01)$ en el porcentaje del corte pierna y sexo sobre el porcentaje de costilla y de paletas. El porcentaje de pierna fue mayor $(\mathrm{P}<0,05)$ en los genotipos $\operatorname{DrX}(25,4 \pm 3,2 \%)$ y KaX $(24,4 \pm 3,2 \%)$ comparado con el Pb (20,6 $\pm 3,2 \%)$. Mientras que el porcentaje del corte paleta fue más alto $(\mathrm{P}<0,05)$ en machos $(23,9 \pm 0,8$ vs $19,3 \pm 0,8 \%)$ y el de costillas en hembras $(20,5 \pm 0,8$ vs $16,4 \pm 0,8 \%)$, respectivamente.

\section{DISCUSIÓN}

Cuando las combinaciones entre temperatura y humedad relativa producen un ITH $\geq 23$ unidades se consideran condiciones ambientales suficientes para producir un estrés calórico sobre los ovinos (Kelly y Bond 1971). Por los ITH registrados durante el desarrollo del experimento se consideró que los animales estuvieron bajo un estrés calórico de moderado a severo, principalmente en mayo (entre 18,9 y 24,1 unidades) y junio (entre 20,4 y 28,5 unidades). En ovinos y otras especies domésticas, las condiciones de estrés calórico se relacionan con una disminución en el consumo de alimento como consecuencia de una reducción en el funcionamiento de la glándula tiroides (Marai y col 2007). En consecuencia, esta reducción en el consumo se refleja negativamente sobre la tasa de crecimiento, peso al sacrificio y sobre la calidad de la carne (Srikandakumar y col 2003). En este estudio, el consumo diario de alimento, la ganancia diaria de peso y el rendimiento en canal presentaron valores promedios de 1,3 kg, 206 g y 53\%, respectivamente. Estos resultados son semejantes y en ocasiones superiores a otros reportados en corderos Pelibuey puros y cruzados bajo condiciones

Cuadro 3. Medias \pm errores estándar (E.E.) del comportamiento productivo en corderos machos y hembras del genotipo Pelibuey puro y cruzados.

Means \pm standard error of the productive performance in male and female lambs of the genotype Pure Pelibuey and their crosses.

\begin{tabular}{|c|c|c|c|c|c|c|c|}
\hline \multirow{2}{*}{ Variables } & \multicolumn{4}{|c|}{ Genotipo** } & \multicolumn{3}{|c|}{ Sexo } \\
\hline & DrX & $\mathrm{KaX}$ & $\mathrm{Pb}$ & E.E. & Macho & Hembra & E.E. \\
\hline Ganancia diaria de peso $(\mathrm{g} / \mathrm{d})$ & $240,0^{\mathrm{a}}$ & $200,0^{\mathrm{b}}$ & $180,0^{\mathrm{b}}$ & 0,1 & $250,0^{\mathrm{a}}$ & $170,0^{\mathrm{b}}$ & 0,1 \\
\hline Consumo de alimento $(\mathrm{kg} / \mathrm{d})$ & $1,5^{\mathrm{a}}$ & $1,3^{\mathrm{b}}$ & $1,2^{\mathrm{b}}$ & 0,0 & $1,4^{\mathrm{a}}$ & $1,2^{\mathrm{b}}$ & 0,0 \\
\hline Conversión alimenticia ${ }^{*}$ & $6,3^{\mathrm{a}}$ & $6,3^{\mathrm{a}}$ & $6,9^{\mathrm{a}}$ & 0,3 & $5,9^{\mathrm{a}}$ & $7,3^{\mathrm{b}}$ & 0,2 \\
\hline
\end{tabular}

Celdas dentro de genotipo y sexo con diferente superíndice $(\mathrm{a}, \mathrm{b}, \mathrm{c})$ significa diferencia a $\mathrm{P}<0,05$.

* Conversión alimenticia: Consumo de alimento/Ganancia diaria de peso $(\mathrm{kg} / \mathrm{kg})$.

** DrX: Dorper x Pelibuey, KaX: Katahdin x Pelibuey, y Pb: Pelibuey puro. 
Cuadro 4. Medias \pm errores estándar (E.E.) de características de canal en corderos machos y hembras del genotipo Pelibuey puro y cruzados.

Means \pm standard error of carcass characteristics in male and female lambs of the genotype Pure Pelibuey and their crosses.

\begin{tabular}{|c|c|c|c|c|c|c|c|}
\hline \multirow{2}{*}{ Variables } & \multicolumn{4}{|c|}{ Genotipos* } & \multicolumn{3}{|c|}{ Sexo } \\
\hline & $\operatorname{DrX}$ & $\mathrm{KaX}$ & $\mathrm{Pb}$ & E.E. & Macho & Hembra & E.E. \\
\hline Peso al sacrificio (kg) & $39,9^{\mathrm{a}}$ & $36,6^{\mathrm{b}}$ & $34,2^{\mathrm{c}}$ & 0,8 & $39,4^{\mathrm{a}}$ & $34,4^{\mathrm{b}}$ & 0,6 \\
\hline Peso de canal caliente $(\mathrm{kg})$ & $20,1^{\mathrm{a}}$ & $18,9^{\mathrm{ab}}$ & $18,3^{\mathrm{b}}$ & 0,6 & $20,0^{\mathrm{a}}$ & $18,2^{\mathrm{b}}$ & 0,5 \\
\hline Peso de canal fría (kg) & $18,9^{\mathrm{a}}$ & $17,5^{\mathrm{ab}}$ & $16,6^{\mathrm{b}}$ & 0,6 & $18,8^{\mathrm{a}}$ & $16,5^{\mathrm{b}}$ & 0,5 \\
\hline Rendimiento en canal (\%) & $52,6^{\mathrm{a}}$ & $52,4^{\mathrm{a}}$ & $54,5^{\mathrm{a}}$ & 1,1 & $53,1^{\mathrm{a}}$ & $54,1^{\mathrm{a}}$ & 0,9 \\
\hline Cabeza $(\mathrm{kg})$ & $1,4^{\mathrm{a}}$ & $1,4^{\mathrm{a}}$ & $1,3^{\mathrm{a}}$ & 0,1 & $1,6^{\mathrm{a}}$ & $1,2^{\mathrm{b}}$ & 0,1 \\
\hline Piel (kg) & $2,7^{\mathrm{a}}$ & $2,6^{\mathrm{a}}$ & $2,3^{\mathrm{b}}$ & 0,1 & $2,7^{\mathrm{a}}$ & $2,3^{\mathrm{b}}$ & 0,1 \\
\hline Órganos viscerales (kg) & $1,5^{\mathrm{a}}$ & $1,6^{\mathrm{a}}$ & $1,3^{\mathrm{a}}$ & 0,1 & $1,7^{\mathrm{a}}$ & $1,2^{\mathrm{b}}$ & 0,6 \\
\hline Longitud de la canal $(\mathrm{cm})$ & $63,0^{\mathrm{a}}$ & $58,0^{\mathrm{b}}$ & $60,2^{\mathrm{ab}}$ & 1,3 & $62,3^{\mathrm{a}}$ & $58,7^{\mathrm{b}}$ & 1,0 \\
\hline Área del M.L.D. $\left(\mathrm{cm}^{2}\right)^{* *}$ & $16,2^{\mathrm{a}}$ & $16,8^{\mathrm{a}}$ & $16,3^{\mathrm{a}}$ & 0,9 & $17,0^{\mathrm{a}}$ & $15,9^{\mathrm{a}}$ & 0,7 \\
\hline Grasa dorsal $(\mathrm{cm})$ & $0,3^{\mathrm{a}}$ & $0,3^{\mathrm{a}}$ & $0,4^{\mathrm{a}}$ & 0,1 & $0,3^{\mathrm{a}}$ & $0,4^{\mathrm{a}}$ & 0,1 \\
\hline
\end{tabular}

Celdas dentro de genotipo y sexo con diferente superíndice ${ }^{(\mathrm{a}, \mathrm{b}}$ ) significa diferencia a $\mathrm{P}<0,05$.

* DrX: Dorper x Pelibuey, KaX: Katahdin x Pelibuey, y Pb: Pelibuey puro.

** M.L.D. = Muscle Longissimus dorsi;

Cuadro 5. Medias \pm errores estándar (E.E.) de los cortes primarios en corderos machos y hembras del genotipo Pelibuey puro y cruzados.

Means \pm standard error of wholesale cuts in male and female lambs of genotypes pure Pelibuey and their crosses.

\begin{tabular}{|c|c|c|c|c|c|c|c|}
\hline \multirow{2}{*}{ Variables } & \multicolumn{4}{|c|}{ Genotipos* } & \multicolumn{3}{|c|}{ Sexo } \\
\hline & DrX & $\mathrm{KaX}$ & $\mathrm{Pb}$ & E.E. & Macho & Hembra & E.E. \\
\hline Piernas (\%) & $25,4^{\mathrm{a}}$ & $24,4^{\mathrm{a}}$ & $20,6^{\mathrm{b}}$ & 3,2 & $24,2^{\mathrm{a}}$ & $22,8^{\mathrm{a}}$ & 1,0 \\
\hline Lomo (\%) & $21,9^{\mathrm{a}}$ & $19,3^{\mathrm{a}}$ & $18,1^{\mathrm{a}}$ & 4,7 & $18,7^{\mathrm{a}}$ & $20,9^{\mathrm{a}}$ & 1,7 \\
\hline Costillas (\%) & $16,5^{\mathrm{a}}$ & $19,3^{\mathrm{a}}$ & $19,6^{\mathrm{a}}$ & 3,4 & $16,4^{\mathrm{a}}$ & $20,5^{\mathrm{b}}$ & 0,8 \\
\hline Paletas (\%) & $21,9^{\mathrm{a}}$ & $21,3^{\mathrm{a}}$ & $21,5^{\mathrm{a}}$ & 3,2 & $23,9^{\mathrm{a}}$ & $19,3^{\mathrm{b}}$ & 0,8 \\
\hline Cuello (\%) & $5,1^{\mathrm{a}}$ & $6,1^{\mathrm{a}}$ & $5,5^{\mathrm{a}}$ & 1,3 & $5,4^{\mathrm{a}}$ & $5,7^{\mathrm{a}}$ & 0,5 \\
\hline $\operatorname{PTCP}(\mathrm{kg}) * *$ & $16,4^{\mathrm{a}}$ & $16,0^{\mathrm{a}}$ & $15,3^{\mathrm{a}}$ & 1,7 & $15,9^{\mathrm{a}}$ & $15,9^{\mathrm{a}}$ & 0,4 \\
\hline $\operatorname{RCP}(\%)^{3}$ & $85,7^{\mathrm{a}}$ & $84,3^{\mathrm{a}}$ & $79,9^{\mathrm{a}}$ & 5,2 & $83,2^{\mathrm{a}}$ & $83,5^{\mathrm{a}}$ & 1,7 \\
\hline
\end{tabular}

Celdas dentro de genotipo y sexo con diferente superíndice ${ }^{(\mathrm{a}, \mathrm{b}}$ ) significa diferencia a $\mathrm{P}<0,05$.

* DrX: Dorper x Pelibuey, KaX: Katahdin x Pelibuey, y Pb: Pelibuey puro.

** PTCP: Peso total de los cortes primarios, ${ }^{3} \mathrm{RCP}$ : Rendimiento en cortes primarios.

de estabulación y sin presencia de estrés calórico (Bores y col 2002, Canton y col 2007, Canton y col 2009ª Partida de la Peña y col 2009). Por ejemplo, en condiciones tropicales (Canton y col 2007) reportaron valores promedios para consumo de alimento, ganancia de peso y rendimiento en canal de $1,2 \mathrm{~kg} / \mathrm{d}, 232 \mathrm{~g} / \mathrm{d}$ y $55 \%$, respectivamente, en corderos de genotipos similares a los usados en esta investigación. Mientras que bajo un clima templado Partida de la Peña y col 2009 observaron que corderos $\mathrm{Pb}$ y cruzas de Pelibuey con Suffolk y Dorset consumieron 1,1 kg/d de alimento, incrementaron su peso $175 \mathrm{~g} / \mathrm{d}$ y el promedio de rendimiento en canal fue de $52 \%$. El ITH $\geq 23$ que señala como punto de inicio de estrés calórico en ovinos fue estimado en base a razas de lana (Kelly y Bond 1971), lo cual explica por qué las condiciones ambientales en que se realizó este estudio no afectaron negativamente el crecimiento de los corderos estudiados. Hubiese sido apropiado haber usado un punto de ITH referencial de estrés calórico para ovinos de pelo, sin embargo, no se encontró ningún estudio donde éste se haya estimado. 
Los ovinos de pelo son razas que se desarrollaron en climas tropicales donde las temperaturas y humedades son altas; mientras que las razas de lana siempre se han mantenido en climas fríos. Debido a esto, los ovinos de pelo muestran ser razas más tolerantes al calor y adaptables a diferentes condiciones ambientales (Fitzhugh y Bradford 1983). Esta situación permite hipotetizar respecto al potencial que tendrían los ovinos de pelo (como Pelibuey, Dorper y Katahdin), para sobrevivir y producir en cualquier región de México, particularmente considerando que el lugar donde se desarrolló este estudio es una de las regiones del país con el registro de temperaturas más elevadas durante el verano.

Los resultados de efecto de genotipo y sexo sobre el peso final coinciden con los encontrados en otro estudio (Partida de la Peña y col 2009), en el cual reportaron pesos finales similares entre machos de los genotipos $\mathrm{Pb}$, Suffolk x Pelibuey y Dorset x Pelibuey, asimismo, menor peso en las corderas $\mathrm{Pb}$ que en las cruzadas. También en otro experimento Canton y col $2009^{\mathrm{b}}$ observaron que corderos machos de genotipo $\mathrm{Pb}(30,3 \mathrm{~kg})$, DrX (33,8 kg) y KaX $(34,8 \mathrm{~kg})$ presentaron pesos similares después de mantenerlos en corral por $75 \mathrm{~d}$. Estos resultados sugieren un crecimiento similar entre los machos de los tres genotipos, lo cual posiblemente se deba al nivel de proteína $(19,6$ y $16,4 \%)$ y energía $(2,6$ y $2,7 \mathrm{Mcal} / \mathrm{kg}$ ) que contenían la dietas ofrecidas. En un estudio realizado en el centro de México, Ruiz y col (2009) observaron que cuando ofrecieron a corderos Dorper y $\mathrm{Pb}$ una dieta con $18 \%$ de proteína, después de $70 \mathrm{~d}$ de consumo el peso vivo fue similar (37,4 vs $36,8 \mathrm{~kg})$; mientras que cuando ofrecieron la dieta con $14 \%$ de proteína, los corderos Dorper $(37,2 \mathrm{~kg}$ ) pesaron $7 \%$ más que los $\mathrm{Pb}(34,6 \mathrm{~kg})$. Comparando tres niveles de energía metabólica $(2,2,2,5$ y 2,8 Mcal/kg de MS) sobre el comportamiento productivo de corderos $\mathrm{Pb}$, DrX y KaX, se observó un mayor crecimiento cuando se alimentaron con la dieta que contenía 2,8 Mcal/kg de MS, en comparación de cuando se alimentaron con la dieta que contenía 2,2 Mcal/kg de MS (Canton y col 2009a). Por lo tanto, los machos de los tres genotipos de este estudio pesaron estadísticamente igual porque los contenidos de proteína y energía metabolizable estuvieron muy cercanos a los óptimos recomendados en la literatura, ya que según Solís y col (1991) para corderos de crecimiento moderado éstos corresponden a 2,6 Mcal/ kg de MS de EM y 14,7\% de PC. Adicionalmente, las corderas $\mathrm{Pb}$ y KaX presentaron pesos finales inferiores a los corderos de los tres genotipos y corderas DrX, lo cual está relacionado parcialmente con la menor velocidad de crecimiento que presentan las hembras debido a su sistema hormonal (Bores y col 2002). Asimismo, dentro del grupo de hembras, el mayor incremento de peso final en las DrX puede atribuirse parcialmente a que las hembras de raza cárnicas tienden fijar hasta $40 \%$ mayor grasa corporal que las razas prolíficas (Canton y col 2009b). También este resultado se puede deber a la habilidad que tienen los ovinos de genotipo Dorper para madurar a edad más temprana y fijar entre la carne gran cantidad de grasa, lo cual no ha sido observado en ninguna otra raza de pelo, incluyendo Katahdin y Pelibuey (Cloete y col 2007). Lo anterior explica la razón por la que tanto machos como hembras de genotipo DrX presentaron mayores pesos finales en comparación a $\mathrm{Pb}$ y $\mathrm{KaX}$.

En la literatura existe marcada discrepancia concerniente al comportamiento productivo que presentan los corderos producto de esquemas de cruzamiento entre razas de pelo prolíficas y razas especializadas para producción de carne. Algunos estudios (Pineda y col 1998, Bunch y col 2004, Partida de la Peña y col 2009) señalan que los corderos híbridos presentan un mayor incremento en su peso que los puros, mientras en otros no reportan diferencias significativas, sólo tendencias numéricas (Canton y col 2007, Canton y col 2009'a). Los resultados de este estudio coinciden en parte con aquellas investigaciones donde han encontrado diferencias en la tasa de crecimiento entre genotipos a favor de las cruzas. Los corderos DrX ganaron $25 \%$ más de peso por día que los $\mathrm{Pb}$, sin embargo, los corderos KaX presentaron similar tasa de crecimiento que los $\mathrm{Pb}$. Posiblemente estos resultados estén relacionados con el efecto de heterosis (Bunch y col 2004), el cual se expresa en las crías al aparearse progenitores de dos razas puras, aunque en esta investigación la falta de información sobre la ganancia diaria de peso que alcanzan los corderos puros Dorper y Katahdin no permite observar el nivel de heterosis adquirido por la acción del cruzamiento practicado. Otra causa de estos resultados es la diferencia en el peso adulto entre las razas utilizadas (Notter y col 2004). El ovino Pelibuey se caracteriza por ser una raza ligera en relación a la raza Dorper y Katahdin, asimismo, en general a las razas de lana (Bradford 2002).

En el consumo diario de alimento, los corderos DrX consumieron 16 y $18 \%$ más que los $\mathrm{KaX}$ y $\mathrm{Pb}$, respectivamente. En un estudio realizado en Estados Unidos también encontraron que los corderos St. Croix puros consumieron 8 y $16 \%$ menos alimento que las cruzas de cordero Callipyge wool x St. Croix y Dorper x St. Croix, respectivamente (Bunch y col 2004). Igualmente en México han reportado diferencias en el consumo entre corderos $\mathrm{Pb}$, DrX y $\mathrm{KaX}$ de $8 \%$-en promedio- a favor de las cruzas (Canton y col 2009a). En este estudio, los corderos DrX consumieron mayor cantidad de alimento, por lo cual ganaron también mayor PV por día. Se encuentra documentado que los animales cambian su consumo para ajustar sus requerimientos nutricionales (Forbes 1986). Así, el consumo de alimento se relaciona con los cambios en el peso vivo. Cabe mencionar que esta relación positiva entre consumo y ganancia conllevó a que no se observaran diferencias en la conversión alimenticia (6,3 para corderos híbridos y 6,9 para $\mathrm{Pb}$ ). El comportamiento observado entre los genotipos estudiados sobre conversión alimenticia está acorde a lo reportado previamente al comparar corderos de raza de pelo puros y sus cruzas (Canton y 
col 2009a, y Bunch y col 2004). Se puede inferir que los genotipos utilizados presentan similar eficiencia en el uso del alimento consumido al nivel de proteína y energía que contenían las dietas.

Los resultados concernientes al efecto del sexo son consistentes con los reportados en otros estudios (Pineda y col 1998, Bores y col 2002, Partida de la Peña y col 2009) donde mencionan que los machos tienden a presentar tasas de crecimiento más altas y a utilizar más eficientemente el alimento consumido que las hembras. Esto es atribuido al efecto anabólico de los andrógenos presentes en los machos (Bradford 2002). Por otra parte, el mayor consumo de alimento que registraron los machos se relaciona también con el mayor incremento de peso (Bores y col 2002).

En corderos $\mathrm{Pb}$, Suffolk x Pelibuey y Rambouillet x Pelibuey sacrificados a los $35 \mathrm{~kg}$ de PV, no se encontraron diferencias entre las medias del peso de la canal caliente y fría por efecto de genotipo (Gutiérrez y col 2005). Canton y col $\left(2009^{b}\right)$ tampoco encontraron diferencias para estas variables al comparar canales del genotipo $\mathrm{Pb}, \mathrm{DrX}$ y $\mathrm{KaX}$. Ambos resultados son contrarios a los observados en este estudio. Esto posiblemente se deba a que en este trabajo hubo diferencias entre los PV al sacrificio de los corderos (de 10 a 14\% más pesados al sacrificio los DrX y KaX) debido a que fueron sacrificados a la misma edad, mientras que en los resultados reportados en la literatura, los genotipos usados se sacrificaron a pesos similares. Además, canales de DrX fueron más pesadas y largas comparadas a las de $\mathrm{Pb}$ debido a diferencias en el tamaño corporal en relación al peso maduro (Bradford 2002). Sin embargo, estas diferencias entre genotipos no fueron detectadas en la evaluación de rendimiento de canal, lo que es coincidente con lo reportado por otros autores (Bores y col 2002, Gutiérrez y col 2005, Partida de la Peña y col 2009). La ausencia de diferencias en rendimiento de canal entre los genotipos estudiados se atribuye a que no se observaron variaciones significativas sobre el espesor de grasa dorsal (Bradford 2002). Aunque existen otros factores que también pueden ayudar a explicar este resultado, tal como el bajo contenido gastrointestinal al sacrificio, grado de finalización y los pesos similares registrados para los componentes corporales no considerados en el peso de la canal (Gutiérrez y col 2005). Por otra parte, el peso de la piel varió entre los genotipos siendo más pesadas las de corderos DrX $(2,7 \mathrm{~kg})$ y $\mathrm{KaX}(2,6 \mathrm{~kg})$. Esto se debe al mayor tamaño corporal que presentaban dichos genotipos. Características como peso de la cabeza, peso de los órganos viscerales, área del músculo Longissimus dorsi y el espesor de grasa dorsal no mostraron variaciones en sus medias por efecto de genotipo. Igualmente, otros autores tampoco reportaron diferencias para estas características de canal entre corderos $\mathrm{Pb}$ y sus cruzas con razas cárnicas (Bores y col 2002, Bunch y col 2004).

En la literatura se menciona que entre corderos de genotipos de razas puras o sus cruzas no hay diferencias en cortes primarios cuando son expresados como porcentaje del peso de la canal (Snowder y Dunckett 2003, Gutiérrez y col 2005, Espinoza y col 2009). Esto debido a que a medida que se expresa el peso como porcentaje, la variabilidad entre pesos de la canal y de cada corte tiende a disminuir. Los resultados para cortes primarios por efecto de genotipo del presente estudio son consistentes con la literatura a excepción de porcentaje del corte pierna, el cual fue 4,2\% mayor en las cruzas. El incremento del peso de las piernas como porcentaje de canal fría posiblemente esté relacionado con una madurez más tardía en la raza Pelibuey en comparación con los otros genotipos (Espinoza y col 2009), y además, con una mayor cantidad de grasa intramuscular en las piernas de los corderos híbridos, ya que en otro estudio se encontró que la grasa se distribuye más rápidamente en los cuartos anteriores comparado con las otras partes de la canal (Gallo 2002).

Los resultados de efecto de sexo sobre características de la canal se debieron en gran medida a diferencias entre los pesos al sacrificio. Los machos presentaron en promedio, un peso al sacrificio de $39,4 \mathrm{~kg}$, mientras que las hembras de $34,4 \mathrm{~kg}$. Así, variables relacionadas con la longitud y el peso de los componentes de la canal (canal caliente, canal fría, cabeza, piel y órganos viscerales) presentaron valores más altos en machos que en hembras. En un estudio (Gutiérrez y col 2005) donde sacrificaron corderos y corderas a los $35 \mathrm{~kg}$ de los genotipos $\mathrm{Pb}$, Suffolk x Pelibuey y Rambouillet x Pelibuey, no se observaron diferencias en peso $(15,9 \mathrm{~kg}$ para machos y $16,5 \mathrm{~kg}$ para hembras) ni en longitud (60,3 cm para machos y $60,4 \mathrm{~cm}$ para hembras) de la canal. También se considera que estos resultados estuvieron influenciados por el efecto hormonal, el cual en los machos favorece que haya un mayor desarrollo muscular y en las hembras una mayor fijación de grasa (Bradford 2002). Anteriormente, en animales Pelibuey y sus cruzas con Suffolk y Dorset se detectó una mejor relación músculo/grasa en machos $(5,8)$ que en hembras $(3,5)$ (Partida de la Peña y col 2009). Por otra parte, otros estudios (Notter y col 2004, Gutiérrez y col 2005) también han reportado que no existen diferencias importantes entre sexos para rendimiento en canal, área del músculo Longissimus dorsi y espesor de grasa dorsal.

Actualmente no existe una tendencia bien definida del efecto de sexo sobre el porcentaje de cortes primarios. Factores como peso al sacrificio, edad, concentraciones hormonales, ambiente y alimentación se han relacionado con las variaciones en los resultados. En este estudio se observó que el sexo solamente influyó sobre el porcentaje de los cortes paleta y de costillares. No obstante, otro estudio (Gutiérrez y col 2005) encontró que el sexo afectó solamente porcentaje de cuello y lomo.

En las condiciones en que se realizó el presente estudio, los corderos de genotipo DrX presentaron tasas de crecimiento y consumos de alimento más altos que los genotipos $\mathrm{Pb}$ y $\mathrm{KaX}$. Además, a pesar de que los corderos 
$\mathrm{Pb}$ no son de raza cárnica, mostraron rendimientos en canal tan satisfactorios como las cruzas con razas especializadas en producción de carne (Dorper y Katahdin). Por otra parte, los resultados sugieren que sería recomendable engordar machos en lugar de hembras debido a su mayor tasa de crecimiento, eficiencia para aprovechar los nutrientes del alimento para incrementar su peso y por la producción de canales más pesadas. Los registros de ganancia diaria de peso y peso al sacrificio de los corderos, sugieren buena capacidad de adaptación de las razas de pelo utilizadas al clima árido y seco, al menos en condiciones de confinamiento.

\section{RESUMEN}

El objetivo del presente estudio fue evaluar el comportamiento productivo en corral y las características de canal de 36 corderos (machos y hembras) de los genotipos Pelibuey puro, Dorper x Pelibuey y Katahdin $x$ Pelibuey bajo condiciones desérticas del noroeste de México. Después de $85 \mathrm{~d}$ de prueba de comportamiento en corral, se sacrificaron todos los corderos para la evaluación de sus canales. Los corderos Dorper $\mathrm{x}$ Pelibuey presentaron mayor $(\mathrm{P}<0,05)$ ganancia diaria de peso $\mathrm{y}$ consumo diario de alimento que los otros dos genotipos. Sin embargo, entre los tres genotipos no se observaron diferencias $(P>0,05)$ para rendimiento en canal, área del músculo Longissimus dorsi y espesor de grasa dorsal. Los machos presentaron mayor ganancia diaria de peso $(\mathrm{P}<0,01)$ y menor conversión alimenticia que las hembras. Los pesos de la canal caliente y fría fueron mayores $(\mathrm{P}<0,05)$ en machos que en hembras, pero el rendimiento en canal y el espesor de grasa dorsal fueron similares $(\mathrm{P}>0,05)$ entre ambos sexos. El peso total y el rendimiento de cortes primarios fueron similares $(P>0,05)$ entre los genotipos y los sexos. Estos resultados sugieren que la raza Dorper puede ser usada en esquemas de cruzamiento para mejorar la producción de carne ovina en zonas áridas como las del noroeste de México.

\section{AGRADECIMIENTOS}

A la Coordinación de Posgrado e Investigación de la Universidad Autónoma de Baja California, en Mexicali, México, por el financiamiento de este proyecto de investigación.

\section{REFERENCIAS}

Avendaño RL, FD Álvarez, L Molina, JS Saucedo, A Correa. 2004. Engorda de corderos Pelibuey y sus cruzas con Dorper y Katahdin bajo condiciones de estrés calórico. Memorias del XXVIII Congreso Nacional de Buiatría, Michoacán, México, Pp 10-13.

Bores QRF, PA Velázquez, M Heredia. 2002. Evaluación de razas terminales en esquemas de cruza comercial con ovejas de pelo F1. Téc Pec Méx 40, 71-79.

Bradford GE. 2002. Relationships among traits: growth rate, mature size, carcass composition and reproduction. Sheep \& Goat Res J 17, 38-41.

Bunch TD, RC Evans, S Wang, CP Brennand, DR Whittier, BJ Taylor. 2004. Feed efficiency, growth rates, carcass evaluation, cholesterol level and wool sheep and their crosses. Small Ruminant Res 52, 239-245.

Burke JM, JK Apple. 2007. Growth performance and carcass traits of forage-fed hair sheep wethers. Small Ruminant Res 67, 264-270.

Canton GJ, JA Quintal. 2007. Evaluation of growth and carcass characteristics of pure Pelibuey sheep and their cross with Dorper and Katahdin breeds. J Anim Sci 85, 571.

Canton GJ, QR Bores, RJ Baeza, FJ Quintal, RR Santos, CC Sandoval. $2009^{\text {a }}$. Growth and efficiency of pure and F1 Pelibuey lambs crossbred with specialized breeds for production of meat. J Anim Vet $A d v$ 8, 26-32.

Canton GJ, QR Bores, RJ Baeza, FJ Quintal, RR Santos, CC Sandoval. $2009^{\text {b }}$. Energy retention of F1 Pelibuey lambs crossbred with breeds for meat production. J Anim Vet Adv 8, 2655-2661.

Cloete SWP, MA Snyman, MJ Herselman. 2000. Productive performance of Dorper sheep. Small Ruminant Res 36, 119-135.

Cloete JJE, SWP Cloete, JJ Olivier, LC Hoffman. 2007. Terminal crossbreeding of Dorper ewe to Ile France Merino Landsheep and SA mutton Merino sires: Ewe production and lamb performance. Small Ruminant Res 69, 28-35.

El Khidir IA, SA Babiker, SA Shafie. 1998. Comparative feedlot performance and carcass characteristics of Sudanese desert sheep and goats. Small Ruminant Res 30, 47-151.

Espinoza LMT, A Estrada, JJ Portillo, G Ríos, JC Robles. 2009. Efecto del grupo racial de corderos de pelo en el peso y rendimiento de cortes primarios. Memoria de la XXXVII Reunión de la Asociación Mexicana de Producción Animal, Chihuahua, México, Pp 312-315.

Fitzhugh HA, GE Bradford. 1983. Hair Sheep of Western Africa and the Americas: A Genetic Resource for the Tropics. Westview, Boulder, CO, USA.

Forbes JM. 1986. The voluntary food intake of farm animals. Butterworths, London, UK, Pp 205-208.

Gallo C. 2002. Crecimiento y composición de canales. En: Tadich N (ed). Salud y producción ovina. Universidad Austral de Chile, Valdivia, Chile, Pp 165-188.

García E. 1987. Modificaciones al sistema de clasificación climática de Koeppen. Editorial Universidad Nacional Autónoma de México, México, Pp 12-13.

Gutiérrez J, MS Rubio, RD Méndez. 2005. Effects of crossbreeding Mexican Pelibuey sheep with Rambouillet and Suffolk on carcass traits. Meat Sci 70, 1-5.

Kelly CF, TE Bond. 1971. Bioclimatic factors and their measurement: A guide to environmental research on animals. National Academy of Sciences, Washington, DC, USA.

Marai IFM, AA El-Darawany, A Fadiel, MAM Abdel-Hafez. 2007. Physiological traits as affected by heat stress in sheep- A review. Small Ruminant Res 71, 1-12.

Notter DR, AL Swiger, RW Harvey. 1975. Adjustment factors for 90-day lamb weight. J Anim Sci 40, 383-391.

Notter DR, SP Greiner, ML Wahlberg. 2004. Growth and carcass characteristics of lambs sired by Dorper and Dorset rams. J Anim Sci 82, 1323-1328.

Partida de la Peña JA, D Braña, L Martínez. 2009. Desempeño productivo y propiedades de la canal en ovinos Pelibuey y sus cruzas con Suffolk y Dorset. Téc Pec Méx 47, 313-322.

Pineda J, JM Palma, FW Haenlein, MA Galina. 1998. Fattening of Pelibuey hair sheep and crossbreds (Rambouillet-Dorset X Pelibuey) in the Mexican tropics. Small Ruminant Res 27, 263-266.

Ruiz NA, JJ Uribe, JR Orozco, VO Fuentes. 2009. The effect of different protein concentrations in the diet of fattening Dorper and Pelibuey lambs. J Anim Vet Adv 8, 1049-1051.

SAS INSTITUTE. 2004. SAS/STAT: User's guide statistics released $9.12^{\text {th }}$ ed. SAS Institute, Inc. Cary, NC, USA.

Snowder GD, SK Duckett. 2003. Evaluations of South Africa Dorper as a terminal sire breed for growth, carcass, and palatability characteristics. J Anim Sci 81, 368-375.

Solís RG, RA Castellanos, AM Velázquez, FG Rodríguez. 1991. Determination of nutritional requirements of growing hair sheep. Small Ruminant Res 4,115-125.

Srikandakumar A, EH Johson, O Mahgoub. 2003. Effect of heat stress on respiratory rate, rectal temperature and blood chemistry in Omani and Australian Merino sheep. Small Ruminant Res 49, 193-198. 